Journal of Computer Science 8 (8): 1397-1406, 2012

ISSN 1549-3636

(C) 2012 Science Publications

\title{
A Policy Based Scheme for Combined Data Security in Mobile Ad hoc Networks
}

\author{
${ }^{1}$ Kartheesn, L. and ${ }^{2}$ S.K. Srivatsa \\ ${ }^{1}$ Department of Computer Science and Engineering, \\ Sri Chandrasekharendra Saraswathi Viswa Mahavidyalaya (SCSVMV University), \\ Kanchipuram, Tamil Nadu, India \\ ${ }^{2}$ St. Joseph College of Engineering, Chennai, Tamil Nadu, India
}

\begin{abstract}
Problem statement: In Mobile Ad hoc Networks (MANET) routing protocols, we require a network-level or link layer security. Since without appropriate security provisions, the MANETs is subjected to attacks like network traffic, replay transmissions, manipulate packet headers and redirect routing messages. In order to address these needs, a policy based network management system that provides the capability to express network requirements is required. Approach: In this study, we propose a policy based scheme for combined data security which focuses mainly on three policies: Integrity, authentication and Confidentiality. For providing security not only to data, but also for routing information, we calculate the trust indexes of the nodes and the route is selected according to the trust value which improves integrity. Then in order to provide authentication, we propose a Distributed Certificate Authority (DCA) technique in which multiple DCA is required to construct a certificate. Next we propose an RSA based novel encryption mechanism in order to provide Confidentiality among the nodes. Thus, the desired level of security is provided by the system based on the policy of the user by executing the corresponding security modules. Results: By simulation results, we show that this scheme provides a combined data security in MANETs and can be used efficiently. Conclusion: Our proposed combined data security policy provides complete protection for the data in MANET communications.
\end{abstract}

Key words: Mobile Ad hoc Networks (MANET), Distributed Certificate Authority (DCA), Certificate Authority (CA), Policy-Based Network Security (PBNS), Vehicular Ad-Hoc Networks (VANETs)

\section{INTRODUCTION}

MANET: The transient infrastructure less multi-hop wireless network in which there is the random movement of the nodes is known as Mobile Ad-Hoc Network (MANET). The wireless transmission range has been extended in MANETs due to its multi-hop packet forwarding. Compatibility in different scenarios can be achieved and there is no infrastructure support which has been deployed in advance (Kushwah and Saxena, 2011). MANETs form an arbitrary topology since it is a self-configuring network of mobile nodes which are connected by wireless links. Movements of the nodes are random and so the wireless topology of the network cannot be predicted and changes rapidly. In emergency situations like natural disasters, military conflicts and emergency medical situations the ad hoc networks are very much suitable due to its minimal configuration, quick deployment and absence of a central governing authority. In the absence of readily available infrastructure networks and for networks of various sizes, the MANETs are applied to configure quickly and dynamically. Even in Vehicular Ad-Hoc Networks (VANETs) and defense sector, MANETs are applied. For flexible civilian applications such as traffic monitoring and emergency assistance services, direct communication between vehicles can be achieved without the need of a cellular infrastructure (Nouak, 2010).

MANET security: The MANET routing protocol is susceptible to many forms of attacks, in the absence of some form of network-level or link layer security. In the wireless network where there is no security provisions the monitoring of network traffic replay transmissions, manipulate packet headers and redirect

Corresponding Author: Kartheesn, L., Department of Computer Science and Enggenering, Sri Chandrasekharendra Saraswathi Viswa Mahavidyalaya (SCSVMV University), Kanchipuram, Tamil Nadu, India 
routing messages seems to be simple. Maintaining the "physical" security of the transmission media in wired infrastructures and routing protocols is quite harder in practice with MANETs (Garg and Mahapatra, 2009). The route discovery and the data transmission phases of MANET communication needs to be protected in order to provide comprehensive security. Though the correctness of the discovered topology information are guaranteed by the routing protocols, the secure and the uninterrupted delivery of transmitted data is not guaranteed. This is due to the fact the adversaries obey with the route discovery and place themselves on exploiting routes.

This causes random interference with the in-transit data and network operation degradation. The spiteful disruptions of data transmission cannot be rectified using the upper layer mechanisms such as reliable transport protocols or reliable data link and acknowledgement routing which are presently assumed by the MANET routing protocols. While there is no communication between the nodes, the data flow is considered to be undisputed and so the communication nodes are easily betrayed for long periods of time. By protecting and verifying all control and data traffic cryptographic, the security attacks can be contradicted. Appropriate trust relationships need to be established with each and every peer that are transiently associated inclusive of the nodes that forwards their data. Due to denial of service attacks, the cryptographic protection is not feasible and this simple discards the data packets (Mamatha and Sharma, 2010).

Threats in MANET: The security in MANETs is subjected to numerous threats as described below:

- The communication channel is highly insecure in MANETs due to its nature of wireless communication and this also leads to Eavesdropping and masquerading

- Unreceptive control of mobile nodes leads a problem in the node security. Cellular nodes theft has been increased and so the MANET nodes are not secure. The node is negotiated and acts as an unreceptive node

- $\quad$ Node tampering is also caused due to theft and this may interrupt network operations or discharge critical information

- The attacker in the denial of service attack can create additional transmissions or expensive computations which are due to the limited powers in the mobile nodes

- Traditional solutions which are based upon the certification authority and on-line servers cannot be used due to infrastructure-less networks
- The routing protocols become too complex due to the absence of fixed topology. It is quite difficult for securing such type of protocol when unreceptive nodes are present.

Some of the MANET security threats are.

Active attacks:

- Denial of Service (DoS)

- Jamming

- Masquerade

- Fabrication

- Modification

Passive attacks:

- $\quad$ Release of message content

- Traffic Analysis

Apart from the usual threats, achieving security within the ad hoc networking is challenging due to following reasons: Wireless Environment, Absence of Central authority, Selfish Nodes, Dynamic Topology, Limited Computational Capability.

Data security: Data security mainly focuses on the following criteria.

Confidentiality: The information about the data and the routing should be received only by the nodes which are permitted to access the information.

Integrity: Since the information can be corrupted by malicious attacks and benign failure like radio propagation impairment, the data should not be revised during transit.

Authentication: The sender should be correctly identified by the receiver and no other sender can be disguised as the sender.

Availability: Operation of the network is not affected by the DoS attack. Physical jamming, disconnection and malfunction of key management service and routing protocol attacks can be commenced at any layer of the network.

Non-repudiation: In order to detect and isolate the compromised nodes the sender is restricted from false denial of a message.

A policy based network management system which provides the ability to express network requirements is used in order to address the above needs. The network 
administrator is given an ability to specify high-level policies in this approach which are as follows.

The aim of long-term, network-wide configuration needs to be specified, e.g., encryption for all private communications is required.

To trigger the correction of network problems based on policies automatically, an automatic feedback loop is required. This triggers the information reported by monitoring agents.

The policy enforcer automatically enforces the policies once after they were described. To configure, control and reconfigure their network in response to network conditions these capabilities provide military personnel with powerful tools (Singh et al., 2010). The following section describes some of the existing policy based approaches.

Problem identification and proposed a solution: In our proposed work, we will design security policy with the following functions.

For confidentiality: Depending on different application requirements, the payload part may be optionally encrypted with the shared key between the source and the destination.

For authentication: We use a reactive certificate distribution mechanism using multiple Certificate Authority (CA) nodes. Nodes trusting and being trusted by more than one CA should apply for a certificate and private-key-shares from each CA. A node without Certificate or needing to renew his certificate must ask to other nodes in the MANET for a certificate issuing.

For integrity and drop: We use a Trust based packet forwarding scheme for mitigating the data drop attacks. It uses trust values to favor packet forwarding by maintaining incentives and penalties for each node. Each intermediate node marks the packets by adding its hash value and forwards the packet towards the destination node. The destination node checks the incentives and penalties and verifies the hash value for nodes with low incentive and high penalty.

Depending on the nature of data and user requirements, policies with the following choices are held by any user:

- Any one of CONF, AUTH and INTEG

- All of CONF, AUTH and INTEG

- CONF and AUTH

- AUTH and INTEG

- CONF and INTEG where, CONF-confidentiality, AUTH-authentication, INTEG-integrity. The desired level of security is provided by the system based on the policy of the sender and receiver. Hence our proposed security policy can provide complete protection for the data in MANET communications.

Related work: Suresh and Duraiswamy (2011) have proposed a node reputation scheme for reactive routing protocol in MANET security. Their scheme also offers an accep trade-off between delay and uncertainty. Their mobility based node reputation scheme identifies and monitor the node's trustworthiness in sharing the information within the ad hoc network. The proposed reactive schemes offer node authentication and reputation. They also handled mobile nodes information uncertainty with the mobility characteristics and its reputation is evaluated to trust or discard the node's communication.

Alicherry et al. (2009) have introduced a novel distributed security policy enforcement architecture that is designed specifically for MANETs. Their approach harnesses and extends the concept of network capabilities and is especially suited for mobile and heterogeneous communication environments. Their model imposes communication restrictions between MANET nodes by enforcing hop-by-hop policies in a distributed manner.

Singh et al. (2010) have made the traditional approach to security inadequate. With this view in mind decentralized group key management is taken into consideration.. The network considered is not very highly volatile. So we have to investigate the group key management for highly volatile network.

Singh et al. (2010) have proposed a novel structure of the node and each entity holds a secret share SSI of each node in a cluster is controlled by its cluster head, the policy enforcer decides for the working of intelligent agent, which is assigned to do the management, which allows two or more parties to derive shared key as a function of information associated with the protocol and so no party can predetermine the resulting value. The group membership certificate is used for group authentication and by the use threshold key scheme secret data are transferred.

Li et al. (2009) have proposed and developed a policy-based malicious peer detection mechanism, in which context information, such as communication channel status, buffer status and transmission power level, is collected and then used to determine whether the misbehavior is likely a result of malicious activity or not.

Cheng et al. (2010) have presented a Policy-Based Network Security (PBNS) management approach for 
tactical MANETs. This approach leverages the DRAMA policy based network management system and the Smart Firewall system to meet the above requirement. It allows administrators to specify lowlevel network access control policies for each INFOCON level using high-level policies.

Jaisankar et al. (2009) have proposed a novel agent based framework to monitor, detect and isolate misbehaving nodes in the MANET. The proposed framework protects both routing and data forwarding operations, which aiming at improving the efficiency in detecting and isolating misbehaving nodes with a minimum overhead. In their work local neighboring nodes collaboratively monitor each other. A novel honesty rate strategy is introduced in each node to determine the well-behaving nodes.

\section{MATERIALS AND METHODS}

\section{Trust based packet forwarding scheme: Trust index calculation:}

Let:

$\mathrm{Z}=\left\{\mathrm{N}_{1}, \mathrm{~N}_{2}, \ldots . \mathrm{Nn}\right\}$ be the network of nodes.

$\mathrm{T}_{\mathrm{i}}=$ The trust index of node $\mathrm{N}_{\mathrm{i}}$,

$\mathrm{T}_{\text {inc }}=$ The value of trust increment,

$\mathrm{T}_{\mathrm{dec}}=$ The value of trust decrement,

$\mathrm{T}_{\mathrm{th}}=$ The trust threshold value.

$\mathrm{N}_{\mathrm{k}}=$ The node which forwards a data packet $\mathrm{P}_{\mathrm{k}}$

$\mathrm{p}=\mathrm{A}$ positive constant for trust increment and decrement.

\section{Algorithm 1:}

1. Initially, each node maintains a lookup table, which includes sequence numbers, source and destination IP addresses and port numbers and the address of the next hop.

2. Node $\mathrm{N}_{\mathrm{i}}$ receives the data packet $\mathrm{P}_{\mathrm{k}}$.

3. If $P_{k}$ is a retransmitted packet, then

3.1 Nodes i decrements trust index of $\mathrm{N}_{\mathrm{k}}$ by

$$
\mathrm{T}_{\mathrm{dec}}=\mathrm{T}_{\mathrm{dec}} 2 * \mathrm{p}
$$

3.2 Compare $\mathrm{T}_{\mathrm{i}-1}$ with $\mathrm{T}_{\text {th. }}$.

3.3 If $\mathrm{T}_{0}<\mathrm{T}_{\mathrm{i}-1}<\mathrm{T}_{\text {th }}$,

Packet is dropped. Else

The packet is forwarded to node $\mathrm{N}_{\mathrm{i}+1}$.

$\mathrm{N}_{\mathrm{i}}$ updates the lookup table with current trust values. End if

Else

If $P_{k}$ is an acknowledgement packet, then

If $\mathrm{N}_{\mathrm{k}}$ originally forwarded $\mathrm{P}_{\mathrm{k}}$, then

$\mathrm{N}_{\mathrm{i}}$ increment trust index of $\mathrm{N}_{\mathrm{k}}$ by
$\mathrm{T}_{\mathrm{inc}}=\mathrm{T}_{\mathrm{inc}}+\mathrm{p}$
End if
End if

\section{Route selection for integrity: Let:}

- Ti be the Trust index on the individual neighbor

- Ta be the average of the trust index of all the neighbors that forwarded/generated RREP

- Oi be the number of Hops in the route established by the individual node in its RREP, Oa be the average of all Oi's obtained from individual neighbors which forwarded the RREP

- $\mathrm{CRS}_{\mathrm{A}}$ and $\mathrm{CRS}_{\mathrm{B}}$ be the Cost of route selection of the node $\mathrm{A}$ and node $\mathrm{B}$ respectively

- $\operatorname{Tr}(\mathrm{A})$ and $\operatorname{Tr}(\mathrm{B})$ be the trust index of the nodes A and $B$ respectively which represents the trust index of the individual neighbor for a Route

- $\mathrm{N}_{\mathrm{hi}}{ }^{\mathrm{A}}$ and $\mathrm{N}_{\mathrm{hi}}{ }^{\mathrm{B}}$ are the trust index of highest immediate downstream neighbors of the nodes $\mathrm{A}$ and $\mathrm{B}$ respectively

Algorithm 2: The trust index of all the nodes is calculated and then the source node calculates the Cost of route selection (CRS) for all its available routes to the destination using the formula:

$$
\begin{aligned}
& \mathrm{CRS}=(\mathrm{Ti} / \mathrm{Ta}) *(\mathrm{Tr}) *(\mathrm{Oa} / \mathrm{Oi}) \\
& \text { 2. If } \mathrm{CRS}_{\mathrm{A}}=\mathrm{CRS}_{\mathrm{B}} \text {, then } \\
& \text { If } \operatorname{Tr}(\mathrm{A})>\operatorname{Tr}(\mathrm{B}) \text {, then } \\
& \text { Select route } A \text {. } \\
& \text { 2.2 Else if } \operatorname{Tr}(\mathrm{A})=\operatorname{Tr}(\mathrm{B}) \text {, then } \\
& \text { Select } \mathrm{N}_{\text {hi }}{ }^{\mathrm{A}} \\
& \text { 2.3.1 If } \mathrm{N}_{\text {hi }}{ }^{\mathrm{A}}>\mathrm{N}_{\mathrm{hi}}{ }^{\mathrm{B}} \\
& \text { 2.3.2 Else if } \mathrm{N}_{\mathrm{hi}}{ }^{\mathrm{A}}=\mathrm{N}_{\mathrm{hi}}{ }^{\mathrm{B}} \\
& \text { Select the shortest route. } \\
& \text { End if } \\
& \text { End if }
\end{aligned}
$$

\section{Distribution of certificate authority among cluster heads:}

- Initially each node calculates its trust index using the above method. Each node gets the trust value of all its neighboring nodes

- The node with the highest trust value is taken as a cluster head in that particular cluster

- DCA algorithm is proposed, where the DCA private keys are distributed amongst $\mathrm{CHs}$ and become the shareholder DCA nodes

- The $\mathrm{CH}$ can satisfy this role since it holds the positions of responsibility and has direct communication with one another 
- The distribution of the DCA private key is processed and it is maintained among the cluster heads. A shares of the DCA private key need to be issued when a new $\mathrm{CH}$ joins the backbone

- Initially, the node contacts their $\mathrm{CH}$ when a node looks for a DCA service and it then takes up the request with other $\mathrm{CHs}$

We will define our DCA by specifying the following operations:

- System setup or bootstrapping

- Applying a DCA private key

- Joining a new $\mathrm{CH}$

- Evicting an existing $\mathrm{CH}$

- Updating $\mathrm{CH}$ shares

Here, $\mathrm{a}$ and $\mathrm{b}$ are large primes such that $\mathrm{b}$ divides $\mathrm{a}-1$ and $\mathrm{s}$ is a generator of the subgroup $\mathrm{Sb}$ of $\mathrm{Za}$ of order $\mathrm{b}$. The values $\mathrm{a}, \mathrm{b}$ and $\mathrm{s}$ are public system parameters. In addition, let $\mathrm{h}$ be a hash function whose range is $\{1 \ldots b-1\}$.

Bootstrapping: Let, $\mathrm{C}$ be the initial set of $\mathrm{CHs}$ at a system setup time, $|\mathrm{C}|=1$ and $\mathrm{m}$ be the required threshold of co-operation between CHs.

All the $\mathrm{CHs}$ participating in the shared key construction is required for the establishment of a $(\mathrm{m}, \mathrm{l})$ threshold sharing of a private key. In the construction of the NTDR backbone, this CHs participation is just a part. The following Distributed Key Generation (DKG) algorithm is used.

Each CHi chooses $v_{i}$ in Za and calculates $e_{i}=s v_{i}$ mod a.

CHi creates a $(\mathrm{m}, \mathrm{l})$ threshold sharing of the secret value $v_{i}$ by generating a polynomial.

Function $f_{i}(z)=\sum_{i=0}^{r-1} u_{i}, t x^{t}$ of degree at most $r-1$ with $f_{i}$ $(0)=v_{i} \bmod u$.

In order to distribute the subshare $\mathrm{fi}(\mathrm{j})$ to $\mathrm{CHj}, \mathrm{Chi}$ uses a secure unicast channel (i.e) (n-1) secure unicast channels is required by the Chi.

$\mathrm{CHi}$ broadcasts the values ei, $\mathrm{t}=$ svi $\bmod \mathrm{a}$. The consistency of the subshares is verified by these values and are sent by $\mathrm{CHi}$. Let $\mathrm{Et}=\prod \mathrm{i} € \mathrm{C}$ ei,t, where $\mathrm{t} €\{0 \ldots$ .r $\mathrm{r}-1\}$.

Each $\mathrm{CHj}$ verifies that the subshare $\mathrm{fi}(\mathrm{j})$ received from $\mathrm{CHi}$ is valid by checking that:

$$
\begin{aligned}
& r-1 \\
& s^{\mathrm{fi}(j)}=\prod\left(e_{j \mathrm{t}}\right) j^{t} \\
& t=0
\end{aligned}
$$

Only if this condition is satisfied, we consider the value received from $\mathrm{Chi}$ is accurate. Else the other $\mathrm{CHs}$ receives a broadcast message from $\mathrm{CHj}$, a warning that an inconsistent subshare has been received from Chi. Then Chi is excluded if at least $\mathrm{k}$ warnings related to Chi is received.

Take $\mathrm{C} 1$ be the set of consistent $\mathrm{CHs}$ at the end of the last stage. Each $\mathrm{CHj}$ in $\mathrm{C} 1$ computes $\mathrm{dj}=\sum_{\mathrm{ieCl}} \mathrm{fi}(\mathrm{j})$ Each of the consistent $\mathrm{CHj}$ holds a share dj of the DCA private key $\operatorname{PrK}=\sum_{\mathrm{jeCl}} \mathrm{fj}(0)$, at the end of this protocol. $\mathrm{PuK}=\prod_{\mathrm{jeCl} 1} \mathrm{e}_{\mathrm{j}}$ is the DCA public key and it is computed from broadcasts exchanges.

Applying a DCA private key: Delivering a DCA security service by a DCA private key is exhibited here. The DCA private key is not known by any $\mathrm{CH}$ and its construction shouldn't be done during any application. A node where a DCA digitally signs a request REQ is considered. The request is forwarded to the backbone. The share of SK can be used by any other $\mathrm{CH}$ receiving a request, in order to sign the request and to produce a signature share, prior to sending it back to the requesting node. The DCA signature can be constructed on REQ when the node has verified $\mathrm{k}$ signature shares. A threshold signature scheme is used for the accomplishment of this process. A variant of the digital signature standard is presented here.

Let:

$\begin{array}{ll}\mathrm{d} € Z_{\mathrm{b}} & =\text { private key } \\ \mathrm{e} & =\mathrm{s}^{\mathrm{d}} \bmod \mathrm{a}\end{array}$

To sign a message $\phi$, first compute $\eta=h(\phi)$, generate a random number $w € Z_{b}$ and then compute:

$\rho=\left(\mathrm{s}^{\mathrm{w}} \bmod \mathrm{a}\right) \bmod \mathrm{b}$

$\psi=\rho l+w \eta \bmod b$ $\psi)$, as:

We denote the signature on message $\phi$ given by ( $\rho$, $\rho=\left(s^{\psi / \eta} e^{-\rho / \eta} \bmod a\right)(\bmod b)$

The following $(\mathrm{m}, \mathrm{l})$ threshold signature scheme based upon the prior variance of DSS, is assumed here Let $\mathrm{C} 2 \underline{\mathrm{C}} \mathrm{C} 1$ (where $|\mathrm{C} 1| \geq \mathrm{m}$ ) is the set of $\mathrm{CHs}$ available to assist in signing request REQ.

Initially a random value e is distributed. In $\mathrm{C} 2$ the $\mathrm{CHs}$ show an example of the DKG algorithm. Adequate consistent $\mathrm{CHs}$ are assumed and the result is a subset $\mathrm{C} 3$ of consistent CHs. This shows that each Chi $€ \mathrm{C} 3$ has a wi share of a random value w. The following public values exist: 


\section{J. Computer Sci., 8 (8): 1397-1406, 2012}

$\mathrm{M}=\mathrm{S}^{\mathrm{w}} \bmod \mathrm{a}$
$\mathrm{P}=\mu \bmod \mathrm{b}$
$\mathrm{H}_{\mathrm{t}}=\prod_{\mathrm{i \epsilon C} 3} \mathrm{~S}^{\mathrm{hi}, \mathrm{t}}$

where, $\mathrm{t} €\{0, \ldots, \mathrm{r}-1\}$ and $\mathrm{h}_{\mathrm{i}, \mathrm{t}}$ are $\mathrm{CHi}$ 's polynomial coefficients. During this process, the $\mathrm{w}$ is not exposed to any $\mathrm{CH}$.

Compute $\psi_{\mathrm{i}}=\rho \mathrm{d}_{\mathrm{i}}+\varepsilon(\mathrm{REQ}) \mathrm{w}_{\mathrm{i}}$ mod $\mathrm{b}$ in each Chi of $\mathrm{C} 3$ and taking that the $|\mathrm{C} 3|$ must be greater than $\mathrm{m}$, send it to the requesting node.

The consistency of the value is verified using the following equation after receiving each $\delta \mathrm{l}$ :

$$
\left.s^{\Psi_{t}}=(e) \prod^{m-1}(E j)^{t j}\right) \rho_{j=1}\left(\mu \coprod^{m-1}(H j)^{t j}\right)_{j=1}^{\varepsilon(R E Q)}
$$

4. Using Lagrange formula to $\{\psi i\}, \psi$ can be computed by the requesting node:

$$
\psi=\sum \prod_{\mathrm{q} \neq \mathrm{j}_{\mathrm{j}=1}}^{\mathrm{m}} \mathrm{i}_{\mathrm{q}} /\left(\mathrm{i}_{\mathrm{q}}-\mathrm{i}_{\mathrm{j}}\right) \text { for any } \mathrm{CH}_{\mathrm{i} 1}, \ldots \ldots . . \mathrm{CH}_{\mathrm{jk}} \notin \mathrm{C} 3
$$

\section{Share updating:}

Update Initialization: In order to ensure that only one initialization node group is present within the whole system, update initialization is done. This corresponds to finding one CA node forming a group with $\mathrm{t} C \mathrm{CA}$ server nodes. Following measures are taken in order to update initialization:

- An initialization message is broadcasted by the selected $\mathrm{CH}$ to all other $\mathrm{CH}$ in the neighboring clusters. The message carries an update request and the ID of the sending $\mathrm{CH}$

- The probability of multiple initializations is reduced by adopting a backoff scheme. After the random backoff period is over the $\mathrm{CH}$ broadcasts the message

- We determine the length of the backoff period as:

- In the range $[0, \mathrm{CwS}]$, the $\mathrm{CH}$ chooses an integer Wi. Contention window size is denoted by CwSi. Prior to broadcasting of the initialization message in the backoff state, the node waits for $(\mathrm{wSi} \times \mathrm{Ts})$ seconds, where $\mathrm{Ts}=$ Time slot size which is common to all $\mathrm{CHs}$

- $\mathrm{CHi}$ monitors the received messages during the backoff period. The backoff state is terminated on receiving any initialization messages from other $\mathrm{CHs}$ and the node starts preparing for the share updating.

- If the node doesn't receive initialization messages, at the end of the backoff period, the node sends an initialization message which floods among all CHs. The involved overhead is inhibited even for large MANETs, due to that the flooding is restricted only in $\mathrm{CHs}$
The trust value keeps changing and so the cluster head is also changed. By this we can share the updating of the message to all other nodes.

Determination of ts and CwS: The $\mathrm{CH}$ with the largest propagation delay within the MANET is selected as the appropriate Ts. The collision of the initialization messages can be avoided by choosing two CHs whose wSi differ by 1 .

If $\mathrm{CwSi}$ is small, the chance of a collision increases, but it leads to a prolonged backup time when the $\mathrm{CwSi}$ increases. The number of $\mathrm{CH}$ nodes is the suitable choice for $\mathrm{CwSi}$.

It is enviable to prioritize the $\mathrm{CH}$ nodes which have enough CA in its cluster or in neighboring clusters, when $t$ CA server nodes are participating in the derivation of new shares.

Generally, we can divide the contention window $(\mathrm{CwS})$ size into three categories.

$\mathrm{CH}$ nodes having more than $\mathrm{t} \mathrm{CA}$ nodes in the cluster-Wa

$\mathrm{CH}$ nodes having less than $\mathrm{t} \mathrm{CA}$ nodes in the cluster, but more than $t \mathrm{CA}$ nodes plus the CA nodes in the direct neighboring cluster-Wb

The remaining $\mathrm{CH}$ nodes assigned largest $\mathrm{CwS}$ value - Wc

The probability of selecting a $\mathrm{CH}$ increases when the $\mathrm{CHs}$ has more CA nodes in its neighborhood.

Multiple initializations can still be avoided using the collision resolution method:

- An acknowledgement is sent to the receiver when a $\mathrm{CH}$ receives the initialization message.

- The ID of the messages is compared by $\mathrm{CH}$, when a new initialization message is received.

- The node ID is checked for the newer message and if it's a smaller one, then the node sends ACK to the new sender NACK is sent to previous sender.

- After all ACKs are collected from the $\mathrm{CH}$ node the winner is determined.

Update procedure: At the end of update initialization, the winning $\mathrm{CH}$ node is represented as Q. New shares are derived as $\mathrm{Q}$ finds $\mathrm{t} \mathrm{CA}$ nodes.

Update propagation: At least $\mathrm{t}$ servers has updated their shares at the end of the update procedure. The remaining CA nodes in the network are updated by the $t$ updated servers. The share updated is propagated to all the $\mathrm{CA}$ in this phase. The $\mathrm{CH}$ is informed about the update completion when CA finishes the share update process. The data regarding the completion of the information from its local cluster is collected by the $\mathrm{CHs}$ and the neighboring $\mathrm{CHs}$ that has no updated 
information is informed about it. For the update information the informed $\mathrm{CHs}$ sends a request to the CA nodes. Based upon the locating method described above, the CAs will then contact the $\mathrm{CHs}$ for locating $\mathrm{t}$ CAs with new shares.

Providing data confidentiality using encryption: The security scheme consists of RSA key exchange mechanism and a novel encryption mechanism to provide security:

- $\quad$ Each node in the network has its own symmetric key Nkey

- To perform encryption and decryption, each node must know other node's Nkey

- At source, Nkey is encrypted with PUr and transmitted to the destination and destination, decrypts Nkey with PRr, where PUr and PRr are the public and private keys of the receiver, respectively

Encryption: The data $M$ is encrypted using the data specific key and the data specific key is encrypted with Nkey. Then, the sender appends the destination nodes ID and transmits this message to its authenticated neighbors. Initially, source node A creates a Data Key Dkey.

The data is encrypted with Dkey, $\left[\mathrm{E}_{\text {Dkey }}(\mathrm{M})\right]$.

Then the DKey is encrypted with A's Nkey [ $\mathrm{E}_{\mathrm{NkeyA}}$ (Dkey)].

Then, the Destination node's ID is appended to the Cipher text:

$\left[\mathrm{E}_{\text {NkeyA }}(\mathrm{Dkey}),\left[\mathrm{E}_{\text {Dkey }}(\mathrm{M})\right.\right.$, DestID]

Decryption: The plain text message is obtained only if the ID of the node matches and it is considered as the intended recipient. The decryption is performed with Nkey of the sender. In order to obtain the original message the decryption is done with the Dkey. The node again re-encrypts the message if it is not an intended recipient. Re-encryption is done with the neighborhood key and it is transmitted to the authenticated neighbor nodes. Once the destination is determined and the original message is decrypted at the destination, the process is stopped.

In the ad hoc network where more attacks can occur, the encryption of the message by Nkey and Dkey provides more security while data forwarding:

\section{Algorithm 3:}

1. Source node A creates Dkey

2. Data is encrypted with Dkey, $\left[\mathrm{E}_{\text {Dkey }}(\mathrm{M})\right]$

3. Dkey is encrypted with A's Nkey [ $\mathrm{E}_{\text {NkeyA }}$ (Dkey)]

4. DestID is attached with cipher text
$\left[\mathrm{E}_{\text {NkeyA }}\left(\right.\right.$ Dkey), $\left[\mathrm{E}_{\text {Dkey }}(\mathrm{M})\right.$, DestID]

5. If DestID matches with receiving node,

5.1 Decryption is performed. Else

5.2 Original message is decrypted

5.3 Re-encrypts the message

6 . Repeat step 5 until the destination node is found.

Policy based scheme for integrity, authentication and confidentiality: Depending upon the nature of data and user requirements, user policies $(\mathrm{P})$ can be specified which can take the following values:

- I-Only Integrity

- Only Authentication

- C-Only Confidentiality.

- IA-Both Integrity and Authentication.

- IC-Both Integrity and Confidentiality.

- AC-Both Authentication and Confidentiality.

- IAC-Integrity, Authentication and Confidentiality

Based on the policy of the user, the corresponding security module(s) can be executed, as per the following algorithm.

\section{Algorithm 4:}

1. If Policy = "I", then

1.1 Calculate the trust index of all the nodes according to algorithm 1 in section 3.1.1

1.2 Select appropriate routes using the algorithm 2 in section 3.1.2.

2. Else if Policy = "A", then

2.1 DCA private keys are applied to deliver security service according to section 3.2.1

2.2 Share updating is done among the cluster heads according to section 3.2.2.

3. Else if Policy $=$ "C," then

3.1 Encryption and Decryption are done according to the algorithm 3 in section 3.3

4. Else if Policy = "I" and Policy = "A", then

4.1 Calculate the trust index of all the nodes according to algorithm 1 in section 3.1.1.

4.2 Select appropriate routes using the algorithm 2 in section 3.1.2.

4.3 DCA private keys are applied to deliver security service according to section 3.2.1

4.4 Share updating is done among the cluster heads according to section 3.2.2.

5. Else if Policy = "I" and Policy ="C", then

5.1 Calculate the trust index of all the nodes according to algorithm 1 in section 3.1.1

5.2 Select appropriate route using the algorithm 2 in section 3.1.2. 
5.3 Encryption and Decryption are done according to the algorithm 3 in section 3.3.

6. Else if Policy ="A" and Policy ="C", then

6.1 DCA private keys are applied to deliver security service according to section 3.2.1.

6.2 Share updating is done among the cluster heads according to section 3.2.2.

6.3 Encryption and Decryption are done according to the algorithm 3 in section 3.3

7. Else if Policy = "I" and Policy ="A" and Policy ="C", then

7.1 Calculate the trust index of all the nodes according to algorithm 1 in section 3.1.1

7.2 Select appropriate routes using the algorithm 2 in section 3.1.2.

7.3 DCA private keys are applied to deliver security service according to section 3.2.1.

7.4 Share updating is done among the cluster heads according to section 3.2.2.

7.5 Encryption and Decryption are done according to the algorithm 3 in section 3.3

End if

\section{RESULTS AND DISCUSSION}

\section{Simulation results:}

Simulation model and parameters: We use NS2 to simulate our proposed algorithm. In our simulation, the channel capacity of mobile hosts is set to the same value: 2 Mbps. We use the Distributed Coordination Function (DCF) of IEEE 802.11 for wireless LANs as the MAC layer protocol. It has the functionality to notify the network layer about link breakage.

In our simulation, 100 mobile nodes move in a $1500 \times 500 \mathrm{~m}$ region for $50 \mathrm{sec}$ simulation time. We assume each node moves independently with the same average speed. All nodes have the same transmission range of $250 \mathrm{~m}$. In our simulation, the number of attackers varies from 2-10. The simulated traffic is Constant Bit Rate (CBR).

Our simulation settings and parameters are summarized in Table 1.

Performance metrics: We evaluate mainly the performance according to the following metrics.

Average end-to-end delay: The end-to-end-delay is averaged over all surviving data packets from the sources to the destinations.

Average packet delivery ratio: It is the ratio of the number of packets received successfully and the total number of packets transmitted.

Drop: It is the number of packets is dropped during the transmission.
Table 1: Simulation Parameters

\begin{tabular}{ll}
\hline No. of nodes & 50 \\
\hline Area size & $1500 \times 500$ \\
Mac & 802.11 \\
Radio range & $250 \mathrm{~m}$ \\
Simulation time & $50 \mathrm{sec}$ \\
Traffic source & $\mathrm{CBR}$ \\
Packet size & 512 \\
Mobility model & Random way point \\
Attack type & Blackhole \\
No. of attackers & $2,4,6,8$ and 10 \\
Pause time & 5 \\
\hline
\end{tabular}

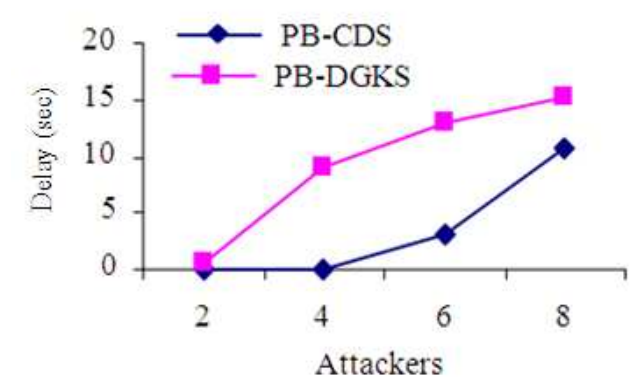

Fig. 1: Attackers Vs delay

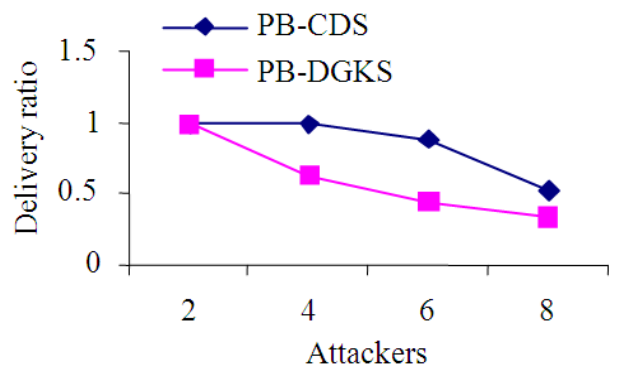

Fig. 2: Attackers Vs delivery ratio

Control overhead: The control overhead is defined as the total number of routing control packets normalized by the total number of receiving data packets.

The simulation results are presented in the next section. We compare our PB-CDS protocol with the PB-DGKS (Singh et al. 2010) protocol in presence of malicious node environment.

Based on attackers: In our experiment, we vary the no. of misbehaving nodes as 2,4,6,8 and 10 .

Figure 1 shows the results of average end-to-end delay for the misbehaving nodes $2,4 \ldots 10$. From the results, we can see that PB-CDS scheme has slightly lower delay than the PB-DGKS scheme because of authentication routines

Figure 2 shows the results of the average packet delivery ratio for the misbehaving nodes $2,4 \ldots . .10$ scenarios. Clearly our PB-CDS scheme achieves more delivery ratio than the PB-DGKS scheme. 


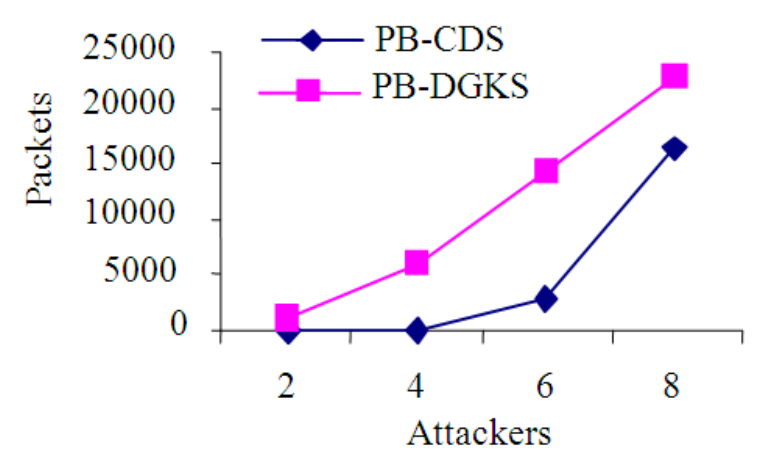

Fig. 3: Attackers Vs drop

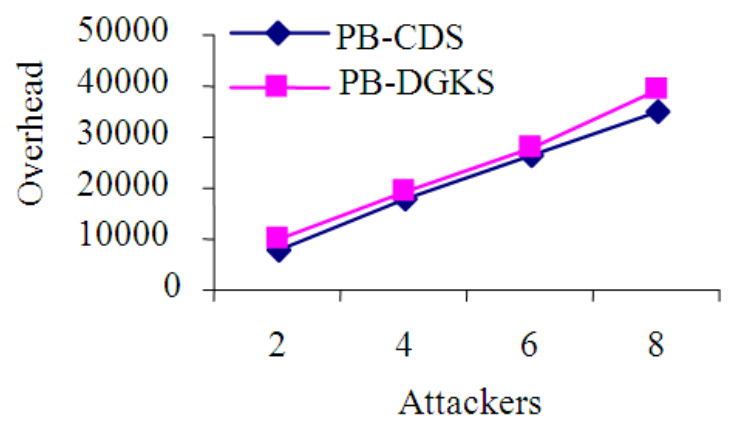

Fig.4: Attackers Vs overhead

Figure 3 shows the results of Packet drop for the misbehaving nodes $2,4 \ldots 10$. From the results, we can see that PB-CDS scheme has less drop than the PBDGKS scheme.

Figure 4 shows the results of routing overhead for the misbehaving nodes $2,4 \ldots 10$. From the results, we can see that PB-CDS scheme has less routing overhead than the PB-DGKS scheme.

\section{CONCLUSION}

In this study we propose a policy based scheme for Combined Data Security which focuses mainly on Integrity, authentication and confidentiality. For providing security not only to data, but also for routing information, we calculate the trust indexes of the nodes and the route is selected according to the trust value. The node with the highest trust value is taken as the source node and the source node calculates the cost of the route selection for all its available routes to the destination. Then in order to provide an Authentication, we propose a Distributed Certificate Authority (DCA) algorithm. Here a DCA private key is distributed among the cluster heads using the threshold signature scheme. Next we propose an RSA key exchange mechanism and a novel encryption mechanism in order to provide Confidentiality among the nodes. In this we use two different symmetric keys to encrypt the message which improves the security while forwarding the data in the ad hoc network. Finally, the user policies can be provided with different probabilities depending upon the nature of the data and the user requirements. From our simulation results we show that this scheme provides a combined data security in MANETs and can be used efficiently.

\section{REFERENCES}

Alicherry, M., A.D. Keromytis and A. Stavrou, 2009. Deny-by-default distributed security policy enforcement in mobile ad hoc networks. Secu. Privacy Commun. Networks, 19: 41-50. DOI: 10.1007/978-3-642-05284-2_3

Cheng, Y.H., A. Ghosh, R. Chadha, M.L. Gary and M. Wolberg, 2010. Managing network security policies in tactical MANETs Using DRAMA. Proceedings of the Military Communications Conference, Oct. 31-Nov. 3, IEEE Xplore Press, USA., $\quad$ pp: $960-964 . \quad$ DOI: 10.1109/MILCOM.2010.5679579

Garg, N. and R.P. Mahapatra, 2009. MANET security issues. J. Comp. Sci. Netw. Secu., 9: 241-246.

Jaisankar, N., R. Saravan and K.D. Swamy, 2009. An agent based security framework for protecting routing layer operations in MANET. Proceedings of the 1st International Conference on Networks and Communications, Dec. 27-29, IEEE Xplore Press, India, pp: 381-385. DOI: 10.1109/NetCoM.2009.64

Kushwah, R.S. and A.S. Saxena, 2011. Reactive multihop routing with MCDs in MANETs. Indian J. Comput. Sci. Eng., 2: 885-891.

Li, W., A. Joshi and T. Finin, 2009. Policy-based malicious peer detection in ad hoc networks. Proceedings of the International Conference on Computational Science and Engineering, Aug. 2931, IEEE Xplore Press, Vancouver, BC., pp: 76-82. DOI: 10.1109/CSE.2009.289

Mamatha, G.S. and S.C. Sharma, 2010. A robust approach to detect and prevent network layer attacks in MANETS. Int. J. Comput. Sci. Secu., 4: 275-284.

Nouak, A., 2010. MANET security. Proceedings of the 6th International conference on intelligent information hiding and multimedia signal processing, Oct. 15-17, Darmstadt, Germany. 
Singh, S., N. Rajpal, A.K. Sharma and R. Pahwa, 2010. Policy based Decentralized group key security for mobile ad-hoc networks. IJCSI Int. J. Comput. Sci. Iss., 7: 44-49.
Suresh, A. and K. Duraiswamy, 2011. Mobile ad hoc network security for reactive routing protocol with node reputation scheme. J. Comput. Sci., 9: 242249. DOI: $10.3844 /$ jcssp. 2011.242 .249 